\title{
Integrin $\beta 4$ and vinculin contained in exosomes are potential markers for progression of prostate cancer associated with taxane-resistance
}

\author{
KYOJIRO KAWAKAMI $^{1}$, YASUNORI FUJITA ${ }^{1}$, TAKU KATO ${ }^{2,3}$, KOSUKE MIZUTANI ${ }^{2}$, KOJI KAMEYAMA ${ }^{2}$, \\ HIROKI TSUMOTO $^{1}$, YURI MIURA ${ }^{1}$, TAKASHI DEGUCHI ${ }^{2}$ and MASAFUMI ITO ${ }^{1}$ \\ ${ }^{1}$ Research Team for Mechanism of Aging, Tokyo Metropolitan Institute of Gerontology, \\ Tokyo 173-0015; Departments of ${ }^{2}$ Urology and ${ }^{3}$ Informative Clinical Medicine, \\ Gifu University Graduate School of Medicine, Gifu, Gifu 501-1193, Japan
}

Received March 5, 2015; Accepted April 14, 2015

DOI: $10.3892 /$ ijo.2015.3011

\begin{abstract}
Treatment with taxanes for castration-resistant prostate cancer often leads to the development of resistance. It has been recently demonstrated that exosomes present in the body fluids contain proteins and RNAs in the cells from which they are derived and could serve as a diagnostic marker for various diseases. In the present study, we aimed to identify proteins contained in exosomes that could be markers for progression and taxane-resistance of prostate cancer. Exosomes were isolated by differential centrifugation from the culture medium of taxane-resistant human prostate cancer PC-3 cells (PC-3R) and their parental PC-3 cells. Isolated exosomes were subjected to iTRAQ-based quantitative proteomic analysis. Exosomes were also isolated from the culture medium by using anti-CD9 antibody-conjugated magnetic beads. Protein expression was knocked down by siRNA transfection followed by analysis of the silencing effects. Proteomic analysis showed that integrin $\beta 4$ (ITGB4) and vinculin (VCL) were upregulated in exosomes derived from PC-3R cells compared to PC-3 cells. The elevation of ITGB4 and VCL was confirmed in exosomes captured by anti-CD9 antibody from the culture medium of PC-3R cells. Silencing of ITGB4 and VCL expression did not affect proliferation and taxane-resistance of PC-3R cells, but ITGB4 knockdown attenuated both cell migration and invasion and VCL knockdown reduced invasion. Our results suggest that ITGB4 and VCL in exosomes could be useful markers for progression of prostate cancer associated with taxane-resistance, providing the basis for development of an exosome-based diagnostic system.
\end{abstract}

Correspondence to: Dr Masafumi Ito, Research Team for Mechanism of Aging, Tokyo Metropolitan Institute of Gerontology, 35-2 Sakae-cho, Itabashi-ku, Tokyo 173-0015, Japan

E-mail: mito@tmig.or.jp

Key words: castration-resistant prostate cancer, taxane-resistance, exosomes, integrin $\beta 4$, vinculin

\section{Introduction}

Prostate cancer is one of the most common male cancers and is the second-leading cause of cancer death among men in the United States (1). Most patients with prostate cancer well respond to androgen deprivation therapy, but $10-20 \%$ of those develop castration-resistant prostate cancer (CRPC) (2). Taxanes, a class of microtubule-targeting anticancer agents such as paclitaxel and docetaxel, have been administered to CRPC patients and docetaxel is currently used as the first-line chemotherapy (3). Docetaxel therapy demonstrated an overall survival benefit for CRPC patients, but there is a finite amount of time before acquiring resistance $(3,4)$.

Development of resistance to anticancer drugs is associated with more malignant and aggressive phenotype and acceleration of tumor growth in various types of cancer including prostate cancer (5-7). Although prostate-specific antigen (PSA) has been commonly used as a marker for tumor growth, there are no clinically useful markers for diagnosing progression and aggressiveness as well as taxane-resistance of prostate cancer. Given that such markers were available, one could select CRPC patients sensitive to docetaxel, avoid administration to drug-resistant patients and also monitor the drug efficacy, minimizing the incidence of adverse effects as well. Furthermore, prediction of progression and prognosis of CRPC patients might be possible.

Exosomes are microvesicles with a diameter of 40-150 nm that are secreted from cells (8). Exosomes contain a variety of biomolecules such as protein, miRNA, RNA and DNA (9). Molecular signature of exosomes in large part reflects that of the cells from which they are derived. Exosomes are present in the body fluids such as blood and urine and therefore expected to serve as a diagnostic marker for various diseases including cancer (10). It has been recently reported that circulating extracellular vesicles (EV) or exosomes in blood can be used for detection of colorectal cancer using the antigen CD147 embedded in cancer-linked EVs, by which the authors paved the way for exosome-based diagnosis (11). It would be of great benefit if progression and aggressiveness as well as drug-resistance of cancer could be diagnosed by examining exosomes 
in the body fluids. This can be especially useful for prostate cancer patients, because serial biopsy of prostate is not usually performed because of the invasiveness and inaccuracy.

In the present study, we aimed to identify proteins contained in exosomes that could be markers for progression and taxane-resistance of prostate cancer. Specifically, we isolated exosomes by differential centrifugation from taxane-sensitive PC-3 cells and taxane-resistant PC-3R cells that were generated from PC-3 cells and subjected them to quantitative proteomic analysis. We identified integrin $\beta 4$ (ITGB4) and vinculin (VCL) as potential markers for progression and taxane-resistance and also confirmed their presence in exosomes captured by anti-CD9 antibody from the culture medium of PC-3R cells. Lastly, we revealed the roles of ITGB4 and VCL in cell migration and invasion of PC-3R cells. Together, we identified ITGB4 and VCL in exosomes as markers for progression and aggressiveness of prostate cancer and possibly for prediction of taxane-resistance.

\section{Materials and methods}

Reagents and antibodies. Paclitaxel was purchased from Sigma-Aldrich (St. Louis, MO, USA). Anti-CD9, anti-Alix and anti-vinculin antibodies were obtained from Santa Cruz Biotechnology (Santa Cruz, CA, USA). Anti- $\beta$-actin, anti-integrin $\beta 4$ and anti-GAPDH antibodies were from Cell Signaling Technology (Danvers, MA, USA). Anti-TSG101 antibody was obtained from Abcam (Cambridge, UK).

Cell culture. Human prostate cancer PC-3 cells were purchased from the American Type Cell Collection (ATCC; Manassas, VA, USA) and cultured in RPMI-1640 medium supplemented with $10 \%$ fetal bovine serum (FBS) in a humidified atmosphere containing 5\% $\mathrm{CO}_{2}$. Taxane-resistant PC-3 cells (PC-3R) that were resistant to both paclitaxel and docetaxel were generated from parental PC-3 cells as previously described (12). In the present study, we used PC-3R cells viable in the presence of $20 \mathrm{nM}$ paclitaxel.

Isolation of exosomes from the cell culture medium by differential centrifugation. The conditioned medium was harvested from cells cultured in advanced RPMI-1640 medium (Life Technologies, Carlsbad, CA, USA). Exosomes were isolated from the medium according to the previously described method with minor modifications (13). Briefly, $1 \times 10^{6}$ cells were seeded on $150-\mathrm{mm}$ dish and the conditioned medium was collected $72 \mathrm{~h}$ later. The medium was centrifuged at $2,000 \mathrm{x}$ g for $10 \mathrm{~min}$ to get rid of cells and the supernatant was centrifuged at $12,000 \times \mathrm{g}$ for $30 \mathrm{~min}$ to remove debris. Lastly, exosomes were pelleted by ultracentrifugation at $110,000 \mathrm{x} \mathrm{g}$ for $70 \mathrm{~min}$, resuspended in PBS and stored at $-80^{\circ} \mathrm{C}$ until use.

Isolation of exosomes by using anti-CD9 antibody-conjugated magnetic beads. Dynabeads M-270 Epoxy magnetic beads (Life Technologies) were conjugated with mouse monoclonal anti-CD9 antibody (BioLegend, San Diego, CA, USA) according to the manufacturer's protocol. The cell culture medium was centrifuged at 2,000 x g for $10 \mathrm{~min}$ and the supernatant was centrifuged at $12,000 \times \mathrm{g}$ for $30 \mathrm{~min}$. After filtration through $0.20 \mu \mathrm{m}$ polyvinylidene difluoride (PVDF) filter, $30 \mathrm{ml}$ of the filtrate were incubated with $1 \mathrm{mg}$ of the anti-CD9 antibody-conjugated beads for $90 \mathrm{~min}$ with rotation at $4^{\circ} \mathrm{C}$. Beads were then washed 3 times with ice-cold PBS, resuspended in RIPA buffer containing protease inhibitor cocktail and stored at $-20^{\circ} \mathrm{C}$ until use.

Western blot analysis. Whole cell lysates were prepared by resuspending cells in ice-cold lysis buffer [1\% Igepal CA-630, $1 \%$ sodium deoxycholate, $0.1 \%$ SDS, $150 \mathrm{mM} \mathrm{NaCl}, 25 \mathrm{mM}$ Tris- $\mathrm{HCl}(\mathrm{pH} 7.6)]$ containing protease inhibitor cocktail. Cell lysates and exosomes were subjected to electrophoresis on $7.5 \%$ SDS-polyacrylamide gels and transferred to PVDF membranes (GE Healthcare, Little Chalfont, UK). After blocking in $5 \%$ skim milk, membranes were probed with a primary antibody and subsequently with a horseradish peroxidase-linked secondary antibody. After washing, bound proteins were visualized using the ECL Prime Western blotting detection system (GE Healthcare).

Transmission electron microscopic analysis. After fixation with $2.5 \%$ glutaraldehyde and post-fixation with $2 \%$ osmium tetroxide, exosomes were embedded in polymeric resin Quetol 812 (Nisshin EM, Tokyo, Japan) and sectioned by ultramicrotomy into 80-nm thick slices, which were deposited onto $\mathrm{Cu}$ grids. Exosomes were then stained with uranyl acetate and lead citrate and examined with the transmission electron microscope (Hitachi H-7500; Hitachi, Tokyo, Japan).

Particle size analysis. Size distribution analysis of exosomes was performed with the qNano system (Izon Science, Christchurch, New Zealand). After dilution in PBS, the size of exosomes was measured using the NP100 nanopores (particle size analysis range; 70-200 nm) and CPC100 calibration particles and analyzed by the Izon Control Suite 2.2 software according to the manufacturer's instructions.

iTRAQ-based labeling. Exosomes were labeled with iTRAQ reagents using the iTRAQ multiplex kit (AB Sciex, Redwood City, CA, USA) according to the manufacturer's protocol. In brief, exosomes were isolated from two independent cell cultures for PC-3 and PC-3R cells. Exosomes (5 $\mu \mathrm{g})$ diluted in PBS was denatured by SDS, reduced by Tris (2-carboxyethyl) phosphine (TCEP) at $60^{\circ} \mathrm{C}$ for $1 \mathrm{~h}$ and alkylated by cysteine blocking reagent methyl methanethiosulfonate (MMTS) at room temperature for $10 \mathrm{~min}$. Samples were then trypsinized at $37^{\circ} \mathrm{C}$ for $5 \mathrm{~h}$ and labeled at room temperature for $1 \mathrm{~h}$ with iTRAQ reagent 114, 115, 116 or 117. Samples from PC-3 cells were labeled with 114 or 115 and those from PC-3R cells were labeled with 116 or 117 . Labeled samples were cleaned up by using an ICAT cation exchange column (AB Sciex) and desalted using a MonoSpin C18 column (GL Sciences, Tokyo, Japan). After evaporation and reconstitution in $0.1 \%$ trifluoroacetic acid (TFA), samples were subjected to LC-MALDI-TOF/TOF analysis.

LC-MALDI-TOF/TOF analysis. Peptides were separated and automatically spotted onto a MALDI plate using direct nanoLC and MALDI fraction system DiNa-MaP (KYA Technologies, Tokyo Japan). Solvent A was 2\% MeCN/0.1\% TFA and solvent $\mathrm{B}$ was $70 \% \mathrm{MeCN} / 0.1 \%$ TFA. The matrix 
solution was prepared at a concentration of $4 \mathrm{mg} / \mathrm{ml} \alpha$-cyano4-hydroxycinnamic acid (CHCA) and $0.08 \mathrm{mg} / \mathrm{ml}$ ammonium citrate in $70 \% \mathrm{MeCN} / 0.1 \%$ TFA. After sample was loaded onto a trap column (HiQ sil C18W-3, 0.5x1 mm; KYA Technologies), valve was switched and peptides were separated by an analytical column (HiQ sil C18W-3, 0.1 x50 mm; KYA Technologies) at a flow rate of $300 \mathrm{nl} / \mathrm{min}$. The LC gradient was as follows: $0-2 \mathrm{~min}, 0-5 \%$ solvent $\mathrm{B} ; 2-60 \mathrm{~min}$, 5-45\% solvent B; 60-75 min, 45-100\% solvent B; 75-85 min, $100 \%$ solvent B; $85-100$ min, $0 \%$ solvent $\mathrm{B}$. The matrix solution was pumped at a flow rate of $1400 \mathrm{nl} / \mathrm{min}$. The column end was connected directly to the spotter needle. LC eluent was mixed with matrix solution and spotted onto a MALDI plate at 30-sec intervals between 5-90 min for a total of 171 spots. Mass spectra were acquired using the AB Sciex TOF/TOF 5800 system operated on TOF/TOF Series Explorer software version 4.1 (AB Sciex). For each spot, MS spectra were acquired in positive ion mode between $\mathrm{m} / \mathrm{z} 800$ and 4000 and accumulated from 800 laser shots in a randomized raster. MS/MS spectra were acquired using parameters and methods as follows: collision energy, $1 \mathrm{kV}$; CID control, OFF; laser shots, 4000; minimum S/N filter, 50; mininum mass, $800 \mathrm{Da}$; maximum mass, $4000 \mathrm{Da}$; acquisition order/fraction, strongest precursors first; maximum precursors/fraction, 100; precursor mass window, 200 resolution (FWHM); metastable suppression, $\mathrm{ON}$.

Protein identification. All MS/MS data were submitted to ProteinPilot software version 4.5 (AB Sciex). Search parameters were as follows: sample type, iTRAQ 4plex (peptide labeled); Cys. alkylation, MMTS; digestion, trypsin; instrument, 5800; species, Homo sapiens; ID focus, biological modifications; database, uniprot_sprot_can+iso_20100622+Contams+.fasta (AB Sciex). Protein identification was considered to be correct based on the following selection criteria: protein having at least 2 peptides with an ion score $>95 \%$ confidence; and protein with protein score (ProtScore) $>1.3$ (unused, $\mathrm{P}<0.05$, $95 \%$ confidence). The average fold-change (PC-3R/PC-3) was calculated as an average of 4 pairwise comparisons (116/114, 116/115, 117/114 and 117/115).

Small interfering RNA transfection. Two small interfering RNAs (siRNAs) for human ITGB4 and VCL as well as siGENOME non-targeting siRNA control gene were purchased from Thermo Fisher Scientific (Waltham, MA, USA). siRNAs were transfected into cells at $2 \mathrm{nM}$ using Lipofectamine 2000 transfection reagent (Thermo Fisher Scientific) as previously described (14).

Cell growth assay. The number of viable cells in 12-well plate was determined by trypan blue dye exclusion assay.

Cell viability assay. To determine the cell viability after paclitaxel treatment, WST assay was performed in 96-well plate using a kit obtained from Roche (Basel, Switzerland).

Cell migration assay. Transfected cells were cultured in 6-well plate until confluent. Cell layers were wounded by using a 1-ml tip and cultured for $24 \mathrm{~h}$. The wound area was determined by analyzing the images taken at 0 and $24 \mathrm{~h}$ using Photoshop software (Adobe Systems, San Jose, CA, USA). The migration
A

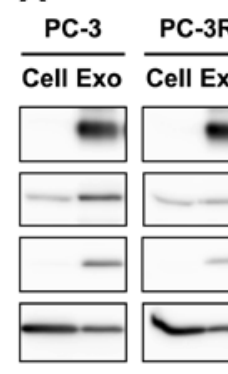

C

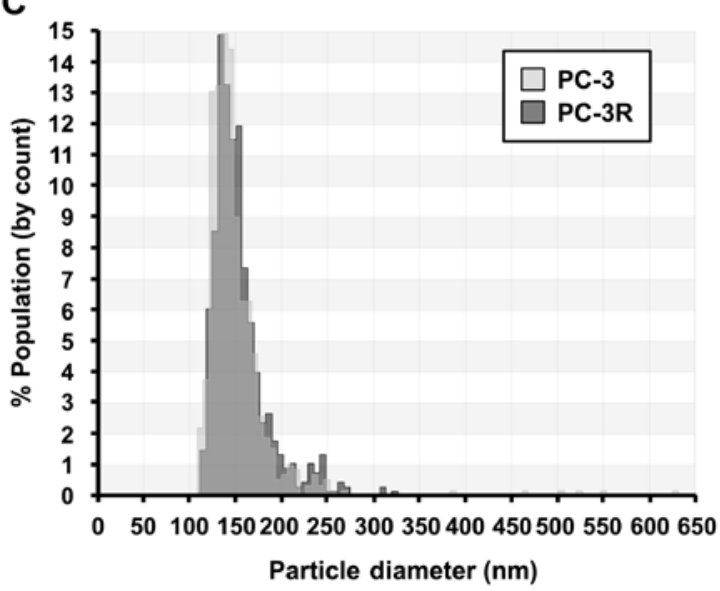

Figure 1. Characterization of exosomes isolated from PC-3R cells and parental PC-3 cells. Exosomes were isolated from the culture medium of PC-3 and PC-3R cells by differential centrifugation. Cell lysates $(5 \mu \mathrm{g})$ and $10 \mu \mathrm{g}$ of exosomes were subjected to western blot analysis for CD9, Alix, TSG101 and GAPDH. Transmission electron microscopic analysis and particle size analysis were also performed for exosomes isolated from both cell lines.

area was determined by subtracting the area free of cells at $24 \mathrm{~h}$ from that at $0 \mathrm{~h}$ and expressed as a percentage of that at $0 \mathrm{~h}$.

Cell invasion assay. Twenty-four hours after seeding $1 \times 10^{5}$ transfected cells onto the Matrigel invasion chamber (Corning Incorp., Corning, NY, USA), cells were fixed with $100 \%$ methanol and stained with crystal violet and the number of invaded cells were counted. The invasion rate was expressed as a percentage of the number of invaded cells in siRNAtransfected cells to that in control siRNA-transfected cells.

Statistical analysis. Statistical differences were determined by the Student's t-test. $\mathrm{P}<0.05$ was considered statistically significant.

\section{Results}

Isolation of exosomes from taxane-sensitive PC-3 and -resistant $P C-3 R$ cells. We isolated exosomes from the cell culture medium by differential centrifugation. In both PC-3 and PC-3R cells, exosomal marker proteins such as CD9, Alix and TSG101 were enriched in exosomes compared to cell lysates and there was no difference in the abundance of the marker proteins between two cell lines (Fig. 1A). The transmission electron microscopic analysis revealed morphology that is typical of exosomes in both PC-3 and PC-3R cells and showed no differ- 
Table I. Proteins upregulated in exosomes isolated from PC-3R cells compared with PC-3 cells.

\begin{tabular}{|c|c|c|c|c|}
\hline Protein name & Gene symbol & Unused cut-off & No. of identified peptides & Fold-change \\
\hline Isoform $\beta$ of $A$-kinase anchor protein 12 & AKAP12 & 8.27 & 4 & 2.58 \\
\hline Vinculin & VCL & 8.00 & 4 & 1.88 \\
\hline Moesin & MSN & 68.84 & 36 & 1.77 \\
\hline Isoform $\beta-4 \mathrm{~B}$ of integrin $\beta-4$ & ITGB4 & 48.33 & 25 & 1.70 \\
\hline
\end{tabular}

Exosomes isolated from PC-3 (labeled with iTRAQ 114 or 115 reporter) and PC-3R (labeled with iTRAQ 116 or 117 reporter) cells were subjected to LC-MALDI-TOF/TOF analysis. The fold-change (PC-3R/PC-3) represents an average of 4 pairwise comparisons (116/114, 116/115, $117 / 114$ and $117 / 115)$.

Table II. Proteins downregulated in exosomes isolated from PC-3R cells compared with PC-3 cells.

\begin{tabular}{|c|c|c|c|c|}
\hline Protein name & $\begin{array}{c}\text { Gene } \\
\text { symbol }\end{array}$ & $\begin{array}{l}\text { Unused } \\
\text { cut-off }\end{array}$ & $\begin{array}{c}\text { No. of identified } \\
\text { peptides }\end{array}$ & Fold-change \\
\hline EGF-like repeat and discoidin I-like domain-containing protein 3 & EDIL3 & 22.00 & 13 & 0.14 \\
\hline Prostaglandin F2 receptor negative regulator & PTGFRN & 9.62 & 6 & 0.35 \\
\hline EH domain-containing protein 4 & EHD4 & 7.76 & 5 & 0.57 \\
\hline Ubiquitin & RPS27A & 8.38 & 4 & 0.63 \\
\hline
\end{tabular}

Exosomes isolated from PC-3 (labeled with iTRAQ 114 or 115 reporter) and PC-3R (labeled with iTRAQ 116 or 117 reporter) cells were subjected to LC-MALDI-TOF/TOF analysis. The fold-change (PC-3R/PC-3) represents an average of 4 pairwise comparisons (116/114, 116/115, $117 / 114$ and $117 / 115)$.

ences in size and shape of exosomes isolated from both cell lines (Fig. 1B). Consistent with these findings, the particle size distribution of exosomes isolated from PC-3 cells was similar to that from PC-3R cells as measured by the qNano particle size analyzer (Fig. 1C). These results indicate that exosomes were successfully isolated by differential centrifugation.

Identification of proteins upregulated in exosomes isolated from $P C$ - $3 R$ cells. It has been previously reported that expression of a variety of genes is altered in taxane-resistant cancer cells including prostate cancer cells, which are involved in taxane-resistance, progression and aggressiveness of cancer (15-17). In order to identify differentially expressed proteins in exosomes between taxane-sensitive PC-3 and -resistant PC-3R cells, we conducted iTRAQ-based quantitative proteomic analysis of exosomes isolated by differential centrifugation. Exosomes were isolated from two independent cell cultures for PC-3 and PC-3R cells. Samples from PC-3 cells were labeled with 114 or 115 reporter and those from PC-3R cells were labeled with 116 or 117 reporter. We detected a total of 110 proteins (data not shown). The fold-changes were $1.00 \pm 0.07$ (mean \pm SD) for PC-3 cells $(115 / 114)$ and $1.01 \pm 0.11$ for PC-3R cells (117/116). The average fold-change (PC-3R/PC-3) was calculated as an average of 4 pairwise comparisons (116/114, 116/115, 117/114 and 117/115). As a result, we identified 4 and 4 proteins upregulated and downregulated in exosomes from PC3-R cells compared with those from PC-3 cells ( $>1.5$-fold and $<0.67$-fold), respectively (Tables I and II). Among upregulated proteins, we selected ITGB4 and VCL for further studies, because integrin and VCL

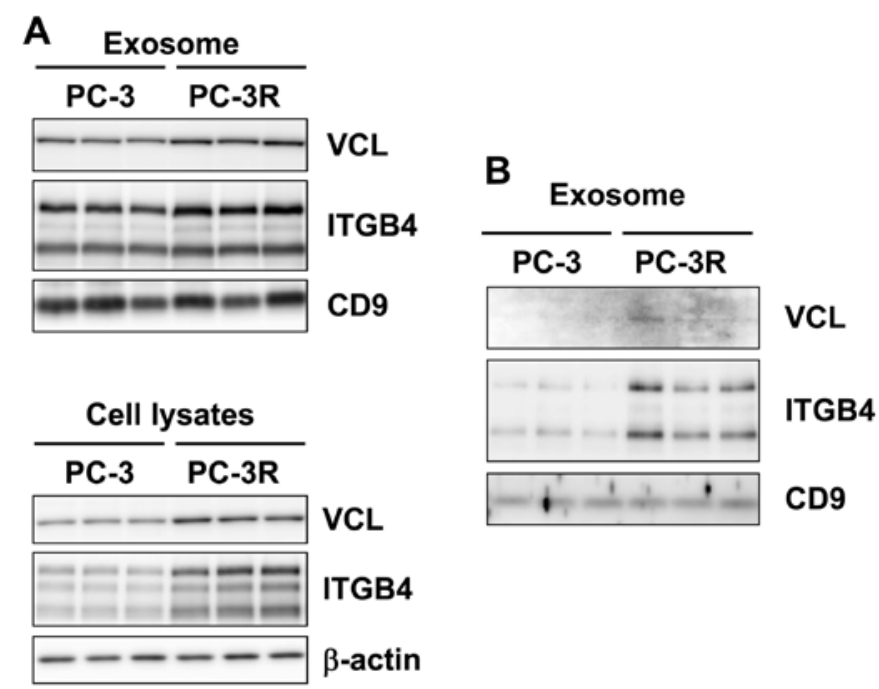

Figure 2. Confirmation of upregulation of ITGB4 and VCL in exosomes isolated from PC-3R cells. (A) Exosomes and cell lysates were isolated from the culture medium of PC-3 and PC-3R cells by differential centrifugation and subjected to western blot analysis for VCL, ITGB4, CD9 and $\beta$-actin. (B) The culture medium $(30 \mathrm{ml})$ of PC-3 and PC-3R cells were incubated with anti-CD9 antibody-conjugated beads $(1 \mathrm{mg})$ at $4^{\circ} \mathrm{C}$ for $90 \mathrm{~min}$. Whole immunocaptured exosomes were subjected to western blot analysis for VCL, ITGB4 and CD9.

are involved in focal adhesion signaling pathways and have been implicated in cancer progression $(18,19)$.

Upregulation of ITGB4 and VCL in exosomes isolated from PC-3R cells by differential centrifugation. In order to 

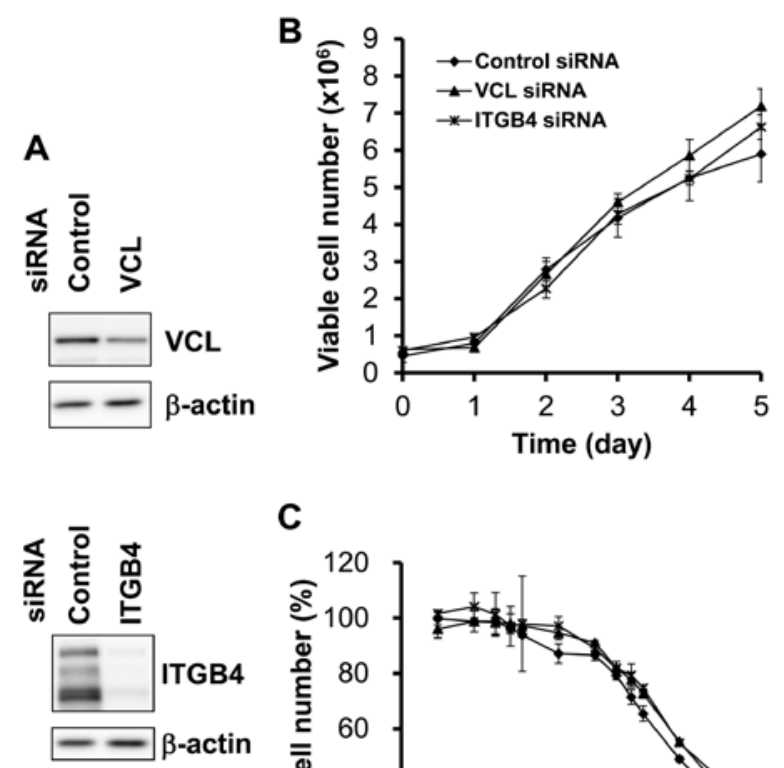

\section{C}

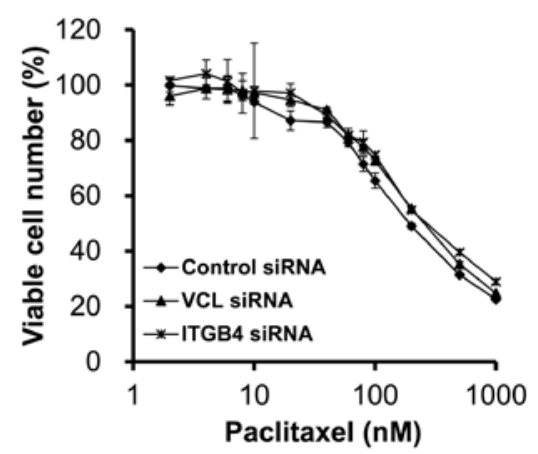

Figure 3. Effects of ITGB4 and VCL knockdown on proliferation and taxane-resistance of PC-3R cells. (A) PC-3R cells were transfected with $2 \mathrm{nM}$ siRNAs and cultured for $48 \mathrm{~h}$ and then cell lysates were harvested and subjected to western blot analysis for VCL, ITGB4 and $\beta$-actin $(5 \mu \mathrm{g})$. (B) Forty-eight hours after siRNA transfection, $5 \times 10^{4}$ cells were replated in 12 -well plate and cultured. Viable cell number was counted at indicated times after seeding by trypan blue dye exclusion assay. (C) Forty-eight hours after siRNA transfection, 3,000 cells were replated in 96-well plate. Cells were treated with paclitaxel for $72 \mathrm{~h}$ at indicated concentrations and WST assay was performed. Data are expressed as mean $\pm \operatorname{SD}(n=3)$.

confirm expression changes, cell lysates as well as exosomes were subjected to western blot analysis. As shown in Fig. 2A, expression of both ITGB4 and VCL was higher in exosomes isolated from PC-3R cells than in those from PC-3 cells. Similarly, ITGB4 and VCL expression was increased in cell lysates of PC-3R cells compared to PC-3 cells. The expression changes were more pronounced in cell lysates than in exosomes. These results suggest that expression of ITGB4 and VCL in exosomes reflected that within cells and that both were elevated in exosomes isolated from PC-3R cells by differential centrifugation.

Upregulation of ITGB4 and VCL in exosomes isolated from $P C-3 R$ cells by using anti-CD 9 antibody-conjugated magnetic beads. In the present study, we isolated exosomes from the cell culture media by using anti-CD9 antibody-conjugated magnetic beads and performed western blot analysis. The results showed that the levels of ITGB4 and VCL in exosomes captured by anti-CD9 antibody from PC-3R cells were higher than those from PC-3 cells, while the level of CD9 in exosomes were similar between two cell lines. It is noteworthy that the difference in the ITGB4 and VCL levels between PC-3 and $\mathrm{PC}-3 \mathrm{R}$ cells was more evident in exosomes isolated by the immunoaffinity method than in those isolated by differential centrifugation. These results suggest that the levels of ITGB4
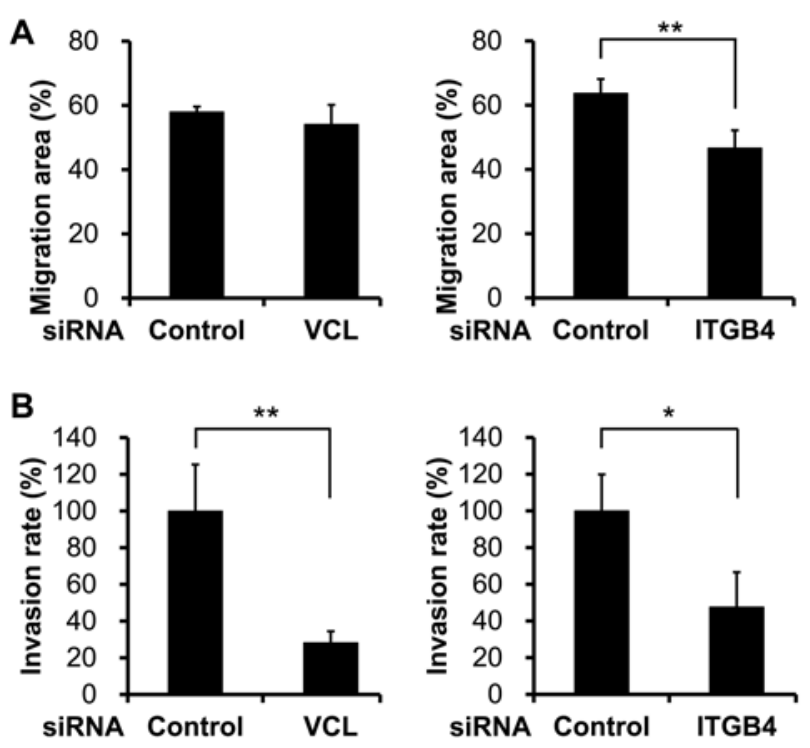

Figure 4. Effects of ITGB4 and VCL knockdown on migration and invasion of PC-3R cells. (A) After transfection with siRNAs, PC-3R cells were cultured until confluent. The cell layer was wounded and cultured for $24 \mathrm{~h}$. Images were taken at time 0 and $24 \mathrm{~h}$. The migration area was determined by subtracting the area free of cells at $24 \mathrm{~h}$ from that at $0 \mathrm{~h}$ and expressed as a percentage of that at $0 \mathrm{~h}$. (B) After transfection with siRNAs, PC-3R cells were re-seeded onto the Matrigel invasion chambers. Twenty-four hours later, invaded cells were fixed, stained and counted. The invasion rate was expressed as a percentage of the number of invaded cells in siRNA-transfected cells to that in control siRNA-transfected cells. Data are expressed as mean $\pm \operatorname{SD}(n=3)$ and evaluated for statistical significance with the Student's t-test $\left({ }^{*} \mathrm{P}<0.05\right.$ and ${ }^{* * *} \mathrm{P}<0.01$, compared with cells transfected with a control siRNA).

and VCL in CD9-positive exosomes from PC-3R cells were elevated compared to those from PC-3 cells.

ITGB4 and VCL knockdown does not affect proliferation and taxane-sensitivity of $P C-3 R$ cells. Although previous studies demonstrated that ITGB4 and VCL play critical roles in various types of cancer including prostate cancer (19-26), their roles in taxane-resistant prostate cancer have not been clarified. We therefore knocked down ITGB4 and VCL expression by siRNA transfection and examined the effect of silencing on proliferation and taxane-resistance. As shown in Fig. 3A, ITGB4 and VCL expression was effectively silenced by siRNAs. There was no difference in proliferation among control siRNA-, ITGB4 siRNA- and VCL siRNA-transfected cells (Fig. 3B). The taxane-resistance was also not affected by ITGB4 and VCL knockdown (Fig. 3C). These result suggest that ITGB4 and VCL that are upregulated in PC-3R cells may not be involved in proliferation and taxane-resistance.

ITGB4 and VCL knockdown reduces migration and/or invasion of PC-3R cells. We next examined if silencing of ITGB4 and VCL expression could influence on migration and invasion of PC-3R cells. As shown in Fig. 4A, the migration activity was decreased in ITGB4 siRNA-transfected cells but not in VCL siRNA-transfected cells compared with control siRNA-transfected cells. In contrast, both ITGB4 and VCL knockdown diminished invasion of PC-3R cells (Fig. 4B). These results suggest that ITGB4 and VCL play an important role in migration and/or invasion of PC-3R cells. 


\section{Discussion}

Drug-resistance is associated with malignant and aggressive phenotype and acceleration of tumor growth in various types of cancer $(5,6)$. In the present study, based on the quantitative proteomic analysis, we found increased levels of ITGB4 and VCL in exosomes isolated from PC-3R cells compared with PC -3 cells. We also confirmed increased expression of both ITGB4 and VCL within PC-3R cells and performed knockdown experiments. The results showed that ITGB4 and VCL play a significant role in migration and/or invasion, but not in proliferation and taxane-resistance. Collectively, we identified ITGB4 and VCL contained in exosomes as potential diagnostic markers for progression and aggressiveness of prostate cancer associated with taxane-resistance.

Integrins are a family of cell surface transmembrane adhesion receptors that connect the actin cytoskeleton to the extracellular matrix (ECM) at focal adhesion and also mediate cell to cell interactions (27). There are $18 \alpha$ and 8 $\beta$ subunits that form 24 integrin heterodimers. The integrin complexes bind to ECM molecules such as fibronectin, collagen and laminin. The $\beta 4$ subunit associates with the $\alpha 6$ subunit forming $\alpha 6 \beta 4$ heterodimer, which mediates integrin signaling activated by laminin. VCL is a cytoskeletal protein that links integrin molecules to the actin cytoskeleton (28). More specifically, VCL binds to F-actin and talin that in turn binds to $\beta$-integrin, by which VCL is involved in cell to cell and cell to ECM interactions. It is intriguing that ITGB4 and VCL that functionally interact and participate in cell adhesion, contractility, motility and growth were identified as exosomal markers for progression and aggressiveness of prostate cancer.

Aberrant expression of integrin subunits has been implicated in the malignant phenotype of a variety of cancer (18). With regard to ITGB4, Banyard et al (29) reported that ITGB4 as well as EPCAM (epithelial cell adhesion molecule) and PLAU [urokinase-type plasminogen activator (uPA)] were elevated in metastatic human prostate cancer DU145-LN4 cells with increased tumorigenesis and increased ability to colonize lymph nodes. The authors also showed that siRNA knockdown of ITGB4 reduced cell migration, but did not inhibit cell invasion into Matrigel. Kumano et al (22) reported that the expression levels of ITGB4 in PC-3 cells were markedly higher compared with those in human prostate cancer LNCaP or DU145 cells and that treatment with AexU may be a useful therapeutic option for prostate cancer when ITGB4 is overexpressed. Yoshioka et al (23) demonstrated that all high-grade prostatic intraepithelial neoplasia (PIN) lesions expressed elevated levels of ITGB4, that ITGB4 was expressed not only in residual basal cells but also in neoplastic cells and that the tumor cells within a large fraction of metastatic lesions exhibited high levels of ITGB4 at the cell surface, suggesting that ITGB4 may play a role in prostate tumor initiation or progression. Increased expression of ITGB4 in pancreatic adenocarcinomas was previously reported by Cruz-Monserrate et al (20). The authors showed that integrin $\alpha 6 \beta 4$ is expressed only on the basal surface of ductal cells in normal pancreas but is dramatically overexpressed during pancreatic adenocarcinoma progression and that it displays altered localization at the earliest stages of pancreatic intraep- ithelial neoplasia (PanIN), thus representing an early event in pancreatic adenocarcinoma progression. These observations support the idea that ITGB4 in exosomes could be a useful marker for progression and also possibly for early detection of cancer.

VCL that links integrin to the actin cytoskeleton was also identified as another exosomal marker for prostate cancer progression in the present study. Dysregulation of VCL expression may result in altered cell adhesion, contractility, motility and growth, all of which are important processes in cancer metastasis (26). In prostate cancer, Ruiz et al (19) found a strong association between VCL gene amplification and overexpression in PC-3 cells. The authors performed immunohistochemical analysis of 443 specimens from across all stages of prostate cancer progression and demonstrated that VCL expression was highest in CRPC, but negative or very low in benign prostatic hyperplasia, suggesting that VCL overexpression might contribute to prostate cancer progression by enhancing tumor cell proliferation. Geisler et al (21) recently reported that VCL expression was significantly upregulated in prostate cancer based on the analysis of prostate tissue samples by 2D-DIGE (two-dimensional difference in gel electrophoresis) and mass spectrometry (MS). Intriguingly, the authors showed that urinary VCL levels in prostate cancer patients were significantly higher than in urine from non-tumor patients. It is tempting to speculate that VCL detected in urine may correspond to VCL contained in exosomes secreted from prostate normal and cancer cells.

We also showed that the ITGB4 and VCL levels in exosomes captured by anti-CD9 antibody-conjugated magnetic beads from PC-3R cells were higher than those from PC-3 cells. The results suggest that ITGB4 or VCL on CD9-positive exosomes could be a marker for progression and invasiveness of prostate cancer. Combined use of anti-ITGB4 or -VCL antibody with anti-CD9 antibody may allow development of exosome-based diagnostic systems. However, it is possible that cells other than cancer cells may secrete CD9-positive exosomes containing ITGB4 or VCL. In order to develop a reliable diagnostic test with good specificity and sensitivity, it is therefore of great importance to detect ITGB4 or VCL in exosomes secreted from the specific cell types of interest. In this regard, we have recently reported that exosomes derived from prostate can be captured from blood by using anti-PSMA (prostate-specific membrane antigen) antibody bound to magnetic beads (30). The detection of ITGB4 or VCL in PSMA-positive exosomes in blood would enable us to develop an accurate diagnostic test for progression and aggressiveness and possibly for early diagnosis and taxane-resistance of prostate cancer. This would also apply to other cancers with increased expression of ITGB4 or VCL, if a reliable surface marker specific to cells such as pancreatic epithelial cells from which exosomes were derived, could be identified.

In conclusion, we have demonstrated that ITGB4 and VCL in exosomes could be potential markers for progression and aggressiveness of prostate cancer associated with taxaneresistance. In order to determine the usefulness of ITGB4 and VCL as exosomal markers for the malignant phenotype of cancer in the clinical setting, development of diagnostic systems and their validation in large-scale clinical studies will be required. 


\section{Acknowledgements}

The present study was supported in part by a Grant-in-Aid from the Ministry of Education, Culture, Sports, Science, and Technology of Japan. The authors are grateful to Mr. Yasuo Hasegawa for his technical support for electron microscopy.

\section{References}

1. Siegel R, Ma J, Zou Z and Jemal A: Cancer statistics, 2014. CA Cancer J Clin 64: 9-29, 2014.

2. Kirby M, Hirst $\mathrm{C}$ and Crawford ED: Characterising the castration-resistant prostate cancer population: A systematic review. Int J Clin Pract 65: 1180-1192, 2011

3. Berthold DR, Pond GR, Soban F, de Wit R, Eisenberger M and Tannock IF: Docetaxel plus prednisone or mitoxantrone plus prednisone for advanced prostate cancer: Updated survival in the TAX 327 study. J Clin Oncol 26: 242-245, 2008.

4. Tannock IF, de Wit R, Berry WR, Horti J, Pluzanska A, Chi KN, Oudard S, Théodore C, James ND, Turesson I, et al; TAX 327 Investigators: Docetaxel plus prednisone or mitoxantrone plus prednisone for advanced prostate cancer. N Engl J Med 351: 1502-1512, 2004.

5. Blagosklonny MV: NCI's provocative questions on cancer: Some answers to ignite discussion. Oncotarget 2: 1352-1367, 2011.

6. Blagosklonny MV: Why therapeutic response may not prolong the life of a cancer patient: Selection for oncogenic resistance. Cell Cycle 4: 1693-1698, 2005.

7. Kim JJ, Yin B, Christudass CS, Terada N, Rajagopalan K, Fabry B, Lee DY, Shiraishi T, Getzenberg RH, Veltri RW, et al: Acquisition of paclitaxel resistance is associated with a more aggressive and invasive phenotype in prostate cancer. J Cell Biochem 114: 1286-1293, 2013.

8. Denzer K, Kleijmeer MJ, Heijnen HF, Stoorvogel W and Geuze HJ: Exosome: From internal vesicle of the multivesicular body to intercellular signaling device. J Cell Sci 113: 3365-3374, 2000.

9. Valadi H, Ekström K, Bossios A, Sjöstrand M, Lee JJ and Lötvall JO: Exosome-mediated transfer of mRNAs and microRNAs is a novel mechanism of genetic exchange between cells. Nat Cell Biol 9: 654-659, 2007.

10. Vlassov AV, Magdaleno S, Setterquist R and Conrad R: Exosomes: Current knowledge of their composition, biological functions, and diagnostic and therapeutic potentials. Biochim Biophys Acta 1820: 940-948, 2012.

11. Yoshioka Y, Kosaka N, Konishi Y, Ohta H, Okamoto H, Sonoda H, Nonaka R, Yamamoto H, Ishii H, Mori M, et al: Ultrasensitive liquid biopsy of circulating extracellular vesicles using ExoScreen. Nat Commun 5: 3591, 2014.

12. Kojima K, Fujita Y, Nozawa Y, Deguchi T and Ito M: MiR-34a attenuates paclitaxel-resistance of hormone-refractory prostate cancer PC3 cells through direct and indirect mechanisms Prostate 70: 1501-1512, 2010.

13. Thery C, Amigorena S, Raposo G and Clayton A: Isolation and characterization of exosomes from cell culture supernatants and biological fluids. Curr Protoc Cell Biol Chapter 3: Unit 3.22, 2006.

14. Fujita Y, Kojima K, Ohhashi R, Hamada N, Nozawa Y, Kitamoto A, Sato A, Kondo S, Kojima T, Deguchi T, et al: MiR-148a attenuates paclitaxel resistance of hormone-refractory, drug-resistant prostate cancer PC3 cells by regulating MSK1 expression. J Biol Chem 285: 19076-19084, 2010.
15. Marín-Aguilera M, Codony-Servat J, Kalko SG, Fernández PL, Bermudo R, Buxo E, Ribal MJ, Gascón P and Mellado B: Identification of docetaxel resistance genes in castration-resistant prostate cancer. Mol Cancer Ther 11: 329-339, 2012.

16. Chang H, Jeung HC, Jung JJ, Kim TS, Rha SY and Chung HC: Identification of genes associated with chemosensitivity to SAHA/taxane combination treatment in taxane-resistant breast cancer cells. Breast Cancer Res Treat 125: 55-63, 2011.

17. Chien AJ and Moasser MM: Cellular mechanisms of resistance to anthracyclines and taxanes in cancer: Intrinsic and acquired. Semin Oncol 35 (Suppl 2): S1-S14, quiz S39, 2008.

18. Desgrosellier JS and Cheresh DA: Integrins in cancer: Biological implications and therapeutic opportunities. Nat Rev Cancer 10: 9-22, 2010.

19. Ruiz C, Holz DR, Oeggerli M, Schneider S, Gonzales IM, Kiefer JM, Zellweger T, Bachmann A, Koivisto PA, Helin HJ, et al: Amplification and overexpression of vinculin are associated with increased tumour cell proliferation and progression in advanced prostate cancer. J Pathol 223: 543-552, 2011.

20. Cruz-Monserrate Z, Qiu S, Evers BM and O'Connor KL: Upregulation and redistribution of integrin alpha6beta4 expression occurs at an early stage in pancreatic adenocarcinoma progression. Mod Pathol 20: 656-667, 2007.

21. Geisler C, Gaisa NT, Pfister D, Fuessel S, Kristiansen G, Braunschweig T, Gostek S, Beine B, Diehl HC, Jackson AM, et al: Identification and validation of potential new biomarkers for prostate cancer diagnosis and prognosis using 2D-DIGE and MS. BioMed Res Int 2015: 454256, 2015.

22. Kumano M, Miyake H, Abolghait SK, Behnsawy HM and Fujisawa M: $\beta 4$-integrin-mediated cytotoxic activity of AexU in human prostate cancer PC3 cells. Oncol Lett 6: 1482-1486, 2013.

23. Yoshioka T, Otero J, Chen Y, Kim YM, Koutcher JA, Satagopan J, Reuter V, Carver B, de Stanchina E, Enomoto K, et al: $\beta 4$ Integrin signaling induces expansion of prostate tumor progenitors. J Clin Invest 123: 682-699, 2013.

24. Gleason B, Adley B, Rao MS and Diaz LK: Immunohistochemical detection of the beta4 integrin subunit in pancreatic adenocarcinoma. J Histochem Cytochem 53: 799-801, 2005.

25. Lipscomb EA and Mercurio AM: Mobilization and activation of a signaling competent alpha6beta4integrin underlies its contribution to carcinoma progression. Cancer Metastasis Rev 24: 413-423, 2005

26. Goldmann WH, Auernheimer V, Thievessen I and Fabry B: Vinculin, cell mechanics and tumour cell invasion. Cell Biol Int 37: 397-405, 2013.

27. Srichai $M$ and Zent R: Integrin structure and function. In: CellExtracellular Matrix Interactions in Cancer. Zent R and Pozzi A (eds). Springer, New York, pp19-41, 2010.

28. Peng X, Nelson ES, Maiers JL and DeMali KA: New insights into vinculin function and regulation. Int Rev Cell Mol Biol 287: 191-231, 2011.

29. Banyard J, Chung I, Migliozzi M, Phan DT, Wilson AM, Zetter BR and Bielenberg DR: Identification of genes regulating migration and invasion using a new model of metastatic prostate cancer. BMC Cancer 14: 387, 2014.

30. Mizutani K, Terazawa R, Kameyama K, Kato T, Horie K, Tsuchiya T, Seike K, Ehara H, Fujita Y, Kawakami K, et al: Isolation of prostate cancer-related exosomes. Anticancer Res 34: 3419-3423, 2014. 